\title{
Metabolic syndrome and the risk of cholangiocarcinoma: a hospital-based case-control study in China
}

This article was published in the following Dove Press journal: Cancer Management and Research

\author{
Jianping Xiong, ${ }^{1, *}$ Xin \\ Lu, ',* Weiyu Xu, ',*Yi Bai,' \\ Hanchun Huang,' Jin Bian,' \\ Lei Zhang,' Junyu Long,' \\ Yiyao Xu, ${ }^{2}$ Zhenjie Wang, ${ }^{2}$ \\ Haitao Zhao' \\ 'Department of Liver Surgery, Peking \\ Union Medical College Hospital, \\ Chinese Academy of Medical Sciences \\ and Peking Union Medical College \\ (CAMS \& PUMC), Beijing, China; \\ ${ }^{2}$ Health Screening Center, Peking \\ Union Medical College Hospital, \\ Chinese Academy of Medical Sciences \\ and Peking Union Medical College \\ (CAMS \& PUMC), Beijing, China \\ *These authors contributed equally \\ to this work
}

Correspondence: Zhenjie Wang Health Screening Center, Peking Union Medical College Hospital, Chinese Academy of Medical Sciences and Peking Union Medical College (CAMS \&PUMC), Beijing 100730, China

Tel +861069156042

Fax +861069156043

Email wangzhenjie2002@aliyun.com

Haitao Zhao

Department of Liver Surgery, Peking

Union Medical College Hospital, Chinese

Academy of Medical Sciences and Peking

Union Medical College (CAMS \&PUMC),

Beijing 100730, China

Tel +861069156042

Fax +861069156043

Email Zhaoht@pumch.cn
Background: Metabolic syndrome is regarded as a risk factor for hepatocellular carcinoma. However, no research has been conducted to investigate the association between metabolic syndrome and cholangiocarcinoma (CCA), especially in the Chinese population. Herein, a hospital-based case-control study was carried out in China to explore the association between metabolic syndrome and CCA risk.

Patients and methods: In this study, 303 CCA patients (136 intrahepatic cholangiocarcinoma [ICC] and 167 extrahepatic cholangiocarcinoma [ECC]) were included, who were observed at Peking Union Medical College Hospital (PUMCH), from 2002 to 2014. Healthy controls were randomly selected from the database of PUMPH Health Screening Center. We retrospectively extracted metabolic syndrome and other possible risk factors from clinical records, followed by investigation of the relationship with CCA via calculation of ORs and 95\% CIs using logistic regression analysis.

Results: Metabolic syndrome was significantly and positively correlated with all CCA subtypes, with adjusted ORs (AORs) of $0.35(95 \% \mathrm{CI}=0.29-0.42)$ and $0.29(95 \% \mathrm{CI}=0.19-0.44)$ for ICC and ECC, respectively (both $P<0.001$ ). Dyslipoproteinemia harbored a stronger relationship with ICC $(\mathrm{OR}=3.16 ; 95 \% \mathrm{CI}=2.12-4.71)$ than $\mathrm{ECC}(\mathrm{OR}=1.87 ; 95 \% \mathrm{CI}=1.27-2.77)$, whereas hypertension harbored a stronger association with ECC $(\mathrm{OR}=3.09 ; 95 \% \mathrm{CI}=2.09-4.58)$ than ICC (OR $=2.06 ; 95 \% \mathrm{CI}=1.32-3.21)$. Obesity was related to both ICC and ECC, with similar ORs, while diabetes was only related to ICC ( $\mathrm{OR}=4.59 ; 95 \% \mathrm{CI}=2.78-7.58)$, but not ECC (OR $=0.97 ;$ 95\% CI $=0.49-1.94)$.

Conclusion: Metabolic syndrome was significantly related to a 1.86-fold elevated CCA risk. Keywords: metabolic syndrome, cholangiocarcinoma, risk factors, case-control

\section{Introduction}

Cholangiocarcinoma (CCA), initially illustrated by Durand Fardel in 1840, is a malignancy originated from the epithelium of bile duct. ${ }^{1,2}$ CCA ranks the second among all primary hepatic carcinoma in terms of incidence, which consists of $3 \%$ of all gastrointestinal malignancies as well as $10-25 \%$ of liver malignancies. ${ }^{3,4}$ Moreover, in recent decades, the incidence of CCC is still rising. Intriguingly, the epidemiology of intrahepatic cholangiocarcinoma (ICC) and extrahepatic cholangiocarcinoma (ECC) varies, with an increasing incidence of the former, but there is a decreasing incidence of the latter in certain regions worldwide, including the UK and the USA. ${ }^{5}$ In the past 20 years, there has been an elevation by $165 \%$ of ICC incidence, but there has been a decline by $14 \%$ of ECC incidence in the USA. ${ }^{6}$ In addition, the prognosis of CCC 
is very poor. The relative 1-, 3- and 5-year survival rates have been revealed to be $25 \%, 9.7 \%$ and $6.8 \%$, respectively, almost without any change in recent decades. ${ }^{7,8}$ In addition, the cause of CCA is not yet fully understood, with only several confirmed risk factors of CCA, including parasitic infections, gallstones, primary sclerosing cholangitis and bile duct cysts. ${ }^{9}$ In recent years, several studies have demonstrated that metabolic syndrome is correlated with CCA. ${ }^{10}$ Metabolic syndrome is composed of a bundle of interrelated metabolic risk factors, including diabetes, obesity, dyslipoproteinemia and hypertension. Besides, the incidence and prevalence of metabolic syndrome are very high, which have been estimated to be as high as $26.7 \%$ in industrialized countries. ${ }^{11}$ However, as a country with a high incidence of CCA, larger hospital-based studies examining the magnitude of this association are lacking in China. Finally, the aim of this study was to explore the relationship between metabolic syndrome and CCA risk in general Chinese population.

\section{Patients and methods}

\section{Study population and design}

A hospital-based case-control study was performed by enrolling 909 subjects (303 subjects with pathologically diagnosed CCA as well as 606 healthy controls) from Peking Union Medical College Hospital (PUMCH) of the Chinese Academy of Medical Sciences and Peking Union Medical College (CAMS \& PUMC), Beijing, China. PUMCH is considered as one of the most common hospitals visited by CCA patients in Beijing.

The patient informatics database of PUMCH was utilized to compile all patients diagnosed with CCA from 2002 to 2014. Patients undergoing potential curative surgery and subsequently diagnosed by pathology were enrolled in this study. CCA was categorized into ICC and ECC. Radiological images (computed tomography [CT], magnetic resonance imaging [MRI] and/or endoscopic retrograde cholangiopancreatography) were reviewed to delineate the anatomic tumor location. ${ }^{12}$ Tumors originated from intrahepatic bile ducts were defined as ICC, while the remaining as ECC. Hilar CCA (ie, Klatskin tumors) is typically considered extrahepatic. ${ }^{13}$

Patients' records were manually and retrospectively reviewed for the extraction of demographical and clinical data as well as risk factors. In this study, we reported demographical data and the detailed information on drinking and smoking. Relevant data were obtained by interviewing patients or their family members, which were simultaneously documented by a physician in a structured data collection sheet. The abovementioned data collection was routinely conducted in our liver surgery clinic, and the relevant sheet was conserved as a part of medical records. Data of age, sex, drinking, smoking, cirrhosis, choledocholithiasis or hepatolithiasis, hepatitis B virus (HBV) and hepatitis C virus (HCV) infection, cholecystolithiasis, fatty liver disease, hypertension, dyslipidemia and diabetes mellitus were abstracted.

Control subjects were recruited from the Health Screening Center of PUMCH, which was not population based; hence, it might be unable to completely reflect the general population. Due to the origin of the case group from PUMCH patients but not from general population, the selection of matched controls from the Health Screening Center of PUMCH rendered the minimum of selection bias. A total of 606 subjects were selected as controls. Cases were frequency matched one to two with controls in the Health Screening Center of PUMCH from May 2012 to July 2017 for age and sex.

\section{Clinical information}

Cirrhosis was histopathologically confirmed or supported by radiographic evidence (portal hypertension, caudate lobe hypertrophy or nodular liver). HBV and HCV infections were confirmed by positive hepatitis B surface antigen and positive HCV RNA, respectively. In addition, diagnosis of $\mathrm{HBV}$ or HCV in physician's note was a confirmation of viral infection. Ever smoker was defined as any person harboring a history of smoking. Hepatolithiasis was diagnosed in the case of stones in intrahepatic duct. Cholecystolithiasis and choledocholithiasis were diagnosed in the existence of stones in gallbladder and common bile duct, respectively. Cystic duct stones were assigned to cholecystolithiasis. Fatty liver disease was diagnosed when a subject had histopathological or radiological evidence of hepatic fatty infiltration with elevated hepatic enzymes.

\section{Definition of metabolic syndrome}

The definition of metabolic syndrome was in line with the US National Cholesterol Education Program Adult Treatment Panel III, which must present with at least three of the following situations: obesity, dyslipidemia, elevated triglycerides, lowered high-density lipoprotein (HDL), hypertension and diabetes. Hypertension was diagnosed according to the standard definition and diagnosis of Commentary on the China Guideline for the Prevention and Treatment of Hypertension in $2004 .{ }^{14}$ The definition of obesity was a body mass index (BMI) of $\geq 25.0 \mathrm{~kg} / \mathrm{m}^{2}$ in accordance with the Asia-Pacific criteria for obesity. ${ }^{15}$ The diagnosis of diabetes was carried out according to the WHO criteria. ${ }^{16}$ In accordance with the National Cholesterol Education Program, the definition 
of dyslipidemia was high-density lipoprotein cholesterol (HDL-C) $<40 \mathrm{mg} / \mathrm{dL}$ as well as Total cholesterol, low-density lipoprotein cholesterol (LDL-C) and TG levels of $\geq 200, \geq 130$ and $\geq 130 \mathrm{mg} / \mathrm{dL}$, respectively. ${ }^{17}$

\section{Statistical analyses}

Age and sex were included as covariates. The Mann-Whitney $U$ test and Pearson chi-squared with Fisher's exact test were utilized for comparison of continuous variables and discrete variables, respectively. Variables with $P$-values of $<0.05$ in the univariate analyses were subsequently adjusted for age and sex to be examined in multiple logistic regression analysis. Logistic regression analysis was conducted for the determination of factors related to CCA progression and calculation of ORs, adjusted ORs (AORs) and 95\% CIs. In addition, the population-attributable risk proportion for statistically significant risk factors was calculated by utilizing the AOR of that factor and its prevalence in the control group. A two-sided $P<0.05$ was considered statistical significance. A SPSS 20.0 (SPSS Inc., Chicago, IL, USA) and SAS statistical software (SAS Institute Inc., Cary, NC, USA) were utilized for statistical analysis.

\section{Ethics approval and informed consent}

This study gained approval from the institutional review board of PUMCH, and all participants provided written informed consent.

\section{Results}

\section{Patient characteristics}

Table 1 summarizes the baseline characteristics of CCA cases and controls. During the study period, 303 CCA cases and 606 controls were enrolled. In the case group, $136 \mathrm{had}$ ICC (44.88\%) and 167 had ECC (55.12\%). In addition, 14 subjects in the ECC group (8.38\%) harbored Klatskin tumor. There were 168 (55.5\%) males of cases and 337 (55.6\%) males of controls. The mean ages were 61.5 and 61.6 years in the case and control groups, respectively. The prevalence of cholecystolithiasis, choledocholithiasis/hepatolithiasis, HBV and $\mathrm{HCV}$ infection, fatty liver disease, cirrhosis, alcohol intake, smoking, diabetes, dyslipoproteinemia, hypertension and obesity were increased in the case group compared to the control group.

\section{Risk factors for CCA}

The results are summarized in Table 2. As expected, the relative odds of CCA were significantly elevated in subjects with a history of cholecystolithiasis (OR $=4.02 ; 95 \% \mathrm{CI}$ $=2.52-6.41 ; P<0.001)$, choledocholithiasis/hepatolithiasis ( $\mathrm{OR}=1.73 ; 95 \% \mathrm{CI}=1.01-2.99 ; P=0.042)$, HCV infection ( $\mathrm{OR}=1.25 ; 95 \% \mathrm{CI}=0.41-3.87 ; P=0.693)$, HBV infection $(\mathrm{OR}=2.63 ; 95 \% \mathrm{CI}=1.54-4.51 ; P<0.001)$, fatty liver disease ( $\mathrm{OR}=2.83 ; 95 \% \mathrm{CI}=1.58-5.08 ; P<0.001)$, alcohol consumption $(\mathrm{OR}=2.01 ; 95 \% \mathrm{CI}=1.42-2.83 ; P<0.001)$ and smoking

Table I Baseline characteristics of CCA cases and controls

\begin{tabular}{|c|c|c|c|c|c|}
\hline Characteristics & CCA, $N=303(\%)$ & ICC, $N=136(\%)$ & $E C C, N=167(\%)$ & Controls, $\mathrm{N}=606(\%)$ & $P$-value \\
\hline \multicolumn{6}{|l|}{ Age (years) } \\
\hline 60 & $136(44.9)$ & $67(49.3)$ & $69(4 I .3)$ & $268(44.3)$ & 0.970 \\
\hline$\geq 60$ & $167(55.1)$ & $69(50.7)$ & $98(58.7)$ & $338(45.7)$ & \\
\hline \multicolumn{6}{|l|}{ Sex } \\
\hline Female & $135(44.6)$ & $75(55.1)$ & $60(35.9)$ & $273(45.1)$ & 0.983 \\
\hline Male & $168(55.4)$ & $61(44.6)$ & $107(64.1)$ & $333(54.9)$ & \\
\hline \multicolumn{6}{|l|}{ Infectious diseases } \\
\hline HBV & $31(10.2)$ & $26(19.1)$ & $5(3.0)$ & $26(4.3)$ & $<0.001$ \\
\hline $\mathrm{HCV}$ & $7(2.3)$ & $5(3.7)$ & $2(1.2)$ & $8(1.3)$ & 0.693 \\
\hline Cholecystolithiasis & $54(17.8)$ & $29(21.3)$ & $25(15.0)$ & $31(5.1)$ & $<0.001$ \\
\hline Choledocholithiasis/hepatolithiasis & $25(8.3)$ & $19(13.9)$ & $6(3.6)$ & $30(0.5)$ & 0.042 \\
\hline Cirrhosis & $19(6.3)$ & $17(12.5)$ & $2(1.2)$ & $28(4.7)$ & $<0.001$ \\
\hline Fatty liver disease & $28(9.2)$ & II (8.I) & $17(10.2)$ & $21(3.4)$ & $<0.001$ \\
\hline Alcohol intake & $76(25.1)$ & $31(22.8)$ & $45(26.9)$ & $88(14.5)$ & $<0.001$ \\
\hline Smoking & III (36.6) & $42(30.9)$ & $69(4 I .3)$ & $105(17.3)$ & $<0.001$ \\
\hline \multicolumn{6}{|l|}{ Metabolic conditions } \\
\hline Diabetes mellitus & $51(16.8)$ & $34(25.2)$ & II (6.6) & $4 I(6.8)$ & $<0.001$ \\
\hline Dyslipoproteinemia & $105(34.7)$ & $56(4 \mid .2)$ & $49(29.3)$ & $108(17.9)$ & $<0.001$ \\
\hline Hypertension & $92(30.4)$ & $35(25.7)$ & $57(34.1)$ & $86(14.2)$ & $<0.001$ \\
\hline Obesity & $62(20.5)$ & $30(22.1)$ & $32(19.2)$ & $71(11.7)$ & 0.001 \\
\hline Metabolic syndrome (overall) & $74(24.4)$ & $39(28.7)$ & $35(21.0)$ & $79(13.1)$ & 0.001 \\
\hline
\end{tabular}

Abbreviations: CCA, cholangiocarcinoma; ECC, extrahepatic cholangiocarcinoma; HBV, hepatitis B virus; HCV, hepatitis C virus; ICC, intrahepatic cholangiocarcinoma. 
Table 2 Logistic regression analysis of risk factors for each CCA subtype

\begin{tabular}{|c|c|c|c|c|c|c|}
\hline \multirow[t]{2}{*}{ Variables } & \multicolumn{2}{|l|}{ CCA, $N=303$} & \multicolumn{2}{|l|}{ ICC, $N=136$} & \multicolumn{2}{|l|}{$E C C, N=167$} \\
\hline & OR (95\% Cl) & $P$-value & OR (95\% Cl) & $P$-value & OR (95\% Cl) & $P$-value \\
\hline \multicolumn{7}{|l|}{ Infectious diseases } \\
\hline HBV & $2.63(1.54,4.5 \mathrm{I})^{\mathrm{a}}$ & $<0.001$ & $5.27(2.95,9.42)^{\mathrm{a}}$ & $<0.001$ & $0.69(0.26,1.82)$ & 0.452 \\
\hline $\mathrm{HCV}$ & $1.25(0.4 \mathrm{I}, 3.87)$ & 0.693 & $2.85(0.92,8.86)$ & 0.070 & $0.91(0.19,4.31)$ & 0.901 \\
\hline Cholecystolithiasis & $4.02(2.52,6.4 I)^{\mathrm{a}}$ & $<0.001$ & $5.03(2.91,8.69)^{\mathrm{a}}$ & $<0.001$ & $3.26(1.86,3.70)^{a}$ & $<0.001$ \\
\hline Choledocholithiasis/hepatolithiasis & $1.73(1.01,2.99)^{\mathrm{a}}$ & 0.042 & $3.12(1.69,5.73)^{\mathrm{a}}$ & $<0.001$ & $3.72(1.19,11.71)$ & 0.024 \\
\hline Cirrhosis & $1.38(0.76,2.52)$ & 0.291 & $2.94(1.56,5.56)^{\mathrm{a}}$ & 0.001 & $0.25(0.06,1.06)$ & 0.250 \\
\hline Fatty liver disease & $2.83(1.58,5.08)^{\mathrm{a}}$ & $<0.001$ & $2.45(I .15,5.2 I)^{\mathrm{a}}$ & $<0.001$ & $3.15(1.62,6.13)^{\mathrm{a}}$ & 0.001 \\
\hline Alcohol intake & $2.0 \mathrm{I}(\mathrm{I} .42,2.83)^{\mathrm{a}}$ & $<0.001$ & $1.74(1.10,2.75)^{\mathrm{a}}$ & 0.019 & $2.17(1.44,3.27)^{\mathrm{a}}$ & $<0.001$ \\
\hline Smoking & $2.76(2.0 \mathrm{I}, 3.78)^{\mathrm{a}}$ & $<0.001$ & $2.13(1.40,3.25)^{\mathrm{a}}$ & $<0.001$ & $3.35(2.31,4.87)^{\mathrm{a}}$ & $<0.001$ \\
\hline \multicolumn{7}{|l|}{ Metabolic conditions } \\
\hline Diabetes mellitus & $2.79(1.80,4.32)^{\mathrm{a}}$ & $<0.001$ & $4.59(2.78,7.58)^{\mathrm{a}}$ & $<0.001$ & $0.97(0.49,1.94)$ & 0.935 \\
\hline Dyslipoproteinemia & $2.39(1.75,3.27)^{\mathrm{a}}$ & $<0.001$ & $3.16(2.12,4.71)^{\mathrm{a}}$ & $<0.001$ & $1.87(1.27,2.77)^{\mathrm{a}}$ & 0.002 \\
\hline Hypertension & $2.64(1.89,3.69)^{\mathrm{a}}$ & $<0.001$ & $2.06(1.32,3.21)^{\mathrm{a}}$ & $<0.001$ & $3.09(2.09,4.58)^{\mathrm{a}}$ & $<0.001$ \\
\hline Obesity & $1.94(1.33,2.82)^{\mathrm{a}}$ & 0.001 & $2.13(1.32,3.43)^{\mathrm{a}}$ & 0.002 & $1.78(1.13,2.82)^{\mathrm{a}}$ & 0.013 \\
\hline Metabolic syndrome (overall) & $1.86(1.29,2.66)^{\mathrm{a}}$ & 0.001 & $2.68(1.72,4.16)^{\mathrm{a}}$ & $<0.001$ & $1.79(1.15,2.79)^{\mathrm{a}}$ & 0.009 \\
\hline
\end{tabular}

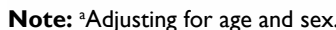

Abbreviations: $\mathrm{CCA}$, cholangiocarcinoma; $\mathrm{ECC}$, extrahepatic cholangiocarcinoma; HBV, hepatitis B virus; HCV, hepatitis C virus; ICC, intrahepatic cholangiocarcinoma.

$(\mathrm{OR}=2.76 ; 95 \% \mathrm{CI}=2.01-3.78 ; P<0.001)$, which were also related to increased CCA risk. We found that $\mathrm{HCV}$ infection $(\mathrm{OR}=1.25 ; 95 \% \mathrm{CI}=0.41-3.87 ; P=0.693)$ and cirrhosis $(\mathrm{OR}$ $=1.38 ; 95 \% \mathrm{CI}=0.76-2.52 ; P=0.291$ ) exerted a neutral effect on CCA risk.

\section{Risk factors for each CCA subtype}

The results are summarized in Table 2 . We conducted a separate analysis of the two subtypes of CCA by pairing controls to identify risk factors for each CCA subtype. As a result, cholecystolithiasis was maintained as the most significant risk factor for both two subtypes, with ORs of 5.03 (95\% $\mathrm{CI}=2.91-8.69)$ and 3.26 (1.86-3.70) for ICC and ECC, respectively $(P<0.001$ for ICC and ECC; Table 2$)$. Choledocholithiasis/hepatolithiasis was also related to an elevated risk of ICC (OR $=3.12 ; 95 \% \mathrm{CI}=1.69-5.73 ; P<0.001)$ and ECC $(\mathrm{OR}=3.72 ; 95 \% \mathrm{CI}=1.19-11.71 ; P=0.024)$. HBV infection was related to an enhanced risk of ICC (OR $=5.27 ; 95 \%$ CI $=2.95-9.42 ; P<0.001)$ but not for ECC $(\mathrm{OR}=0.69 ; 95 \%$ $\mathrm{CI}=0.26-1.82 ; P=0.452)$. HCV infection exerted a neutral effect on the risk of both CCA subtypes. Fatty liver disease rendered a risk for both CCA subtypes, with the largest magnitude in ECC (AOR $=3.15 ; 95 \% \mathrm{CI}=1.62-6.13$ ), followed by ICC (OR $=2.45 ; 95 \% \mathrm{CI}=1.15-5.21)$. Cirrhosis was only positively related to ICC subtype (AOR $=2.94,95 \%$ $\mathrm{CI}=1.56-5.56 ; P<0.001)$, but not overall CCA. The OR of smoking was the highest in ECC $(3.35,95 \% \mathrm{CI}=2.31-4.87$; $P<0.001)$, followed by ICC $(2.13 ; 95 \% \mathrm{CI}=1.40-3.25$; $P<0.001)$. Overall, the abovementioned outcomes implicated that every risk factor rendered risk for each CCA subtype in diverse magnitudes.

\section{Association between metabolic syndrome and CCA risk}

Among the individual situations of metabolic syndrome, diabetes was associated with 2.79-fold elevated CCA risks $(P<0.0001)$. Similarly, obesity, hypertension and dyslipoproteinemia were all significantly $(P<0.0001)$ related to elevated risks, which ranged from 1.94 to 2.39 of developing CCA. A combination of these conditions revealed that CCA patients were significantly more prone to harbor a history of metabolic syndrome than controls (OR $=1.86,95 \% \mathrm{CI}=1.29-2.66$; $P=0.001$; Table 2 ). In the stratification by CCA subtypes, the AORs of metabolic syndrome were consistent for both two CCA subtypes, with ORs of $2.68(95 \% \mathrm{CI}=1.72-4.16)$ and 1.79 (95\% CI $=1.15-2.79)$ for ICC and ECC, respectively. In addition, dyslipoproteinemia was more strongly related to ICC $(\mathrm{OR}=3.16 ; 95 \% \mathrm{CI}=2.12-4.71)$ than $\mathrm{ECC}(\mathrm{OR}=1.87$; $95 \% \mathrm{CI}=1.27-2.77)$, whereas hypertension harbored a stronger correlation with $\mathrm{ECC}(\mathrm{AOR}=3.09 ; 95 \% \mathrm{CI}=2.09-4.58)$ than ICC (AOR $=2.06 ; 95 \% \mathrm{CI}=1.32-3.21)$. Obesity was related to ICC as well as ECC, with similar AORs. Nevertheless, diabetes was only related to ICC ( $\mathrm{OR}=4.59 ; 95 \% \mathrm{CI}$ $=2.78-7.58)$, but not $\mathrm{ECC}(\mathrm{OR}=0.97 ; 95 \% \mathrm{CI}=0.49-1.94)$.

\section{Discussion}

This is the first large hospital-based Chinese study to explore the relationship of metabolic syndrome with CCA 
risk, including ICC and ECC, which indicate that metabolic syndrome is significantly associated with CCA progression. Moreover, cholecystolithiasis, choledocholithiasis/hepatolithiasis, fatty liver disease, HBV infection, HBV infection, alcohol intake and smoking rendered risks of different magnitudes for diverse CCA subtypes. The abovementioned findings are in line with the speculation that ICC and ECC are unique diseases, each of them presenting different susceptibility to risk factors.

$\mathrm{HBV}$ is recognized as a risk factor for hepatocellular carcinoma (HCC). However, the relationships between $\mathrm{HBV}$ and CCA and the effect of HBV in CCA progression remain poorly understood. Similar to the results of previous reports, ${ }^{18,19}$ our findings demonstrate that HBV is strongly related to $\mathrm{CCA}$, which can significantly elevate $\mathrm{CCA}$ risk. The integration of HBV DNA into human chromosomes and the sustained expression of viral proteins, such as HBx proteins and large proteins (LHBs), can activate oncogenes, induce oxidative stress and cause genetic instability. ${ }^{20,21}$ In addition, viruses can cause continuous hepatocyte destruction-regeneration in the inflammatory environments created by the host immune response to infection, thereby creating an environment favorable for genetic mutations leading to hepatocyte transformation..$^{22}$ Besides, a previous study showed that due to the same progenitor cells of cholangiocytes and hepatocytes, HBV may trigger carcinogenesis in cholangiocytes via the same mechanism, which is consistent to the carcinogenesis of hepatocytes. ${ }^{23}$

Cholecystolithiasis, choledocholithiasis/hepatolithiasis, fatty liver disease, alcohol consumption and smoking were related to significantly elevated CCA risk, inconsistent with previous studies. ${ }^{24,25}$ These risk factors have a common feature, ie, they participate in the pathogenesis of CCA by causing chronic biliary tract inflammation, which is a recognized contributor to bile duct carcinogenesis. ${ }^{25}$

Early studies reported an increased prevalence of ICC in patients with metabolic syndrome. ${ }^{10}$ However, a recent study conducted in our center failed to figure out an association between metabolic syndrome and ICC. ${ }^{12}$ Our study confirmed that metabolic syndrome, including diabetes, dyslipoproteinemia, hypertension and obesity, has a positive impact on the risk of CCA. Risk increased by metabolic syndrome is consistent across all two subtypes: 2.68-fold for ICC, 1.79-fold for ECC. Metabolic syndrome is clearly related to nonalcoholic fatty liver disease (NAFLD) and nonalcoholic steatohepatitis (NASH). ${ }^{26,27}$ NAFLD is closely related to $\mathrm{HCC}$ and can sequentially promote the development of liver fibrosis, cirrhosis, end-stage liver disease and eventually HCC. In addition, insulin resistance and subsequent inflammatory cascade are related to NAFLD development. ${ }^{28-31}$ Determining whether NAFLD can also promote CCA in insulin resistance and insulin resistance-related inflammation is difficult because of the low incidence of CCA. However, in consideration of the same progenitor cells of cholangiocytes and hepatocytes, ${ }^{23}$ NAFLD may trigger carcinogenesis in cholangiocytes via the same mechanism. Thus, metabolic syndrome may enhance CCA risk by influencing NAFLD incidence.

Our research has the following strengths. First, this is the first Chinese study to investigate the relationship of metabolic syndrome with CCA risk (including ICC and ECC). We newly identified metabolic syndrome as risk factors for all CCA subtypes. In addition, we explored the role of other common risk factors on CCA incidence as well, including cholecystolithiasis, choledocholithiasis/hepatolithiasis, fatty liver disease, cirrhosis, HBV infection, HCV infection, alcohol intake and smoking. Thus, this is the most comprehensive study with a large sample size (303 CCA patients and 606 controls) to evaluate the possible risk factors for CCA, and its results may be of interest to CCA researchers and of help to clinicians aiming to develop a means of preventing the development of CCA. Second, all CCA patients included in our study were confirmed by pathological and radiological examinations. Therefore, we could avoid ascertainment bias due to misdiagnosis.

However, there are certain limitations in our study. First, the main limitation of this study is its single-center and retrospective design. Despite strict adherence to inclusion and exclusion criteria, selection bias remained as a retrospective study. Second, due to the retrospective property of this study, we were incapable of obtaining detailed information of smoking and alcohol consumption histories, which is likely to help determine the dose-response relationship between smoking, alcohol intake and CCA progression. Moreover, we had no access of data about antiviral treatments in HBV-infected and $\mathrm{HBV}$-infected subjects, which might affect their risk of developing CCA. Third, because of the lack of information about primary sclerosing cholangitis and inflammatory bowel disease in the control group, we cannot estimate the CCA risk related to these factors.

\section{Conclusion}

This hospital-based case-control study indicates metabolic syndrome as a significant risk factor for CCA progression, 
including ICC and ECC. In addition, we also observed that cholecystolithiasis, choledocholithiasis/hepatolithiasis, fatty liver disease, HBV infection, alcohol intake and smoking are also risk factors for CCA progression.

\section{Acknowledgment}

The study was funded by National Key Project Research and Development Projects (S2016G9012), International Science and Technology Cooperation Projects (2015DFA30650) and the Capital Special Research Project for Clinical Application (Z151100004015170).

\section{Author contributions}

All authors contributed toward data analysis, drafting and critically revising the paper and agree to be accountable for all aspects of the work.

\section{Disclosure}

The authors report no conflicts of interest in this work.

\section{References}

1. Olnes MJ, Erlich R. A review and update on cholangiocarcinoma. Oncology. 2004;66(3):167-179.

2. Vijgen S, Terris B, Rubbia-Brandt L. Pathology of intrahepatic cholangiocarcinoma. Hepatobiliary Surg Nutr. 2017;6(1):22-34.

3. Vauthey JN, Blumgart LH. Recent advances in the management of cholangiocarcinomas. Semin Liver Dis. 1994;14(2):109-114.

4. Shaib Y, El-Serag HB. The epidemiology of cholangiocarcinoma. Semin Liver Dis. 2004;24(2):115-125.

5. Taylor-Robinson SD, Toledano MB, Arora S, et al. Increase in mortality rates from intrahepatic cholangiocarcinoma in England and Wales 1968-1998. Gut. 2001;48(6):816-820.

6. Shaib YH, Davila JA, Mcglynn K, El-Serag HB. Rising incidence of intrahepatic cholangiocarcinoma in the United States: a true increase? J Hepatol. 2004;40(3):472-477.

7. Lepage C, Cottet V, Chauvenet M, et al. Trends in the incidence and management of biliary tract cancer: a French population-based study. J Hepatol. 2011;54(2):306-310.

8. Pawlik TM. Intrahepatic cholangiocarcinoma: from diagnosis to treatment. Hepatobiliary Surg Nutr. 2017;6(1):1.

9. Khan SA, Thomas HC, Davidson BR, Taylor-Robinson SD. Cholangiocarcinoma. Lancet. 2005;366(9493):1303-1314.

10. Welzel TM, Graubard BI, Zeuzem S, El-Serag HB, Davila JA, Mcglynn KA. Metabolic syndrome increases the risk of primary liver cancer in the United States: A study in the SEER-Medicare database. Hepatology. 2011;54(2):463-471.

11. Ford ES, Giles WH, Mokdad AH. Increasing prevalence of the metabolic syndrome among u.s. Adults. Diabetes Care. 2004;27(10):2444-2449.

12. Chaiteerakij R, Yang JD, Harmsen WS, et al. Risk factors for intrahepatic cholangiocarcinoma: association between metformin use and reduced cancer risk. Hepatology. 2013;57(2):648-655.
13. Tyson GL, El-Serag HB. Risk factors for cholangiocarcinoma. Hepatology. 2011;54(1):173-184.

14. Giles TD, Materson BJ, Cohn JN, Kostis JB. Definition and classification of hypertension: an update. J Clin Hypertens. 2009;11(11):611-614.

15. Kanazawa M, Yoshiike N, Osaka T, Numba Y, Zimmet P, Inoue S. Criteria and classification of obesity in Japan and Asia-Oceania. World Rev Nutr Diet. 2005;94:1-12.

16. Alberti KG, Zimmet PZ, Definition ZPZ. Definition, diagnosis and classification of diabetes mellitus and its complications. Part 1: diagnosis and classification of diabetes mellitus provisional report of a WHO consultation. Diabet Med. 1998;15(7):539-553.

17. National Cholesterol Education Program (NCEP) Expert Panel on Detection, Evaluation, and Treatment of High Blood Cholesterol in Adults (Adult Treatment Panel III). Third Report of the National Cholesterol Education Program (NCEP) Expert Panel on Detection, Evaluation, and Treatment of High Blood Cholesterol in Adults (Adult Treatment Panel III) final report. Circulation. 2002;106(25): 3143-3421.

18. Palmer WC, Patel T. Are common factors involved in the pathogenesis of primary liver cancers? A meta-analysis of risk factors for intrahepatic cholangiocarcinoma. J Hepatol. 2012;57(1):69-76.

19. Zhou Y, Zhao Y, Li B, et al. Hepatitis viruses infection and risk of intrahepatic cholangiocarcinoma: evidence from a meta-analysis. $B M C$ Cancer. 2012;12:289.

20. Honda M, Yamashita T, Ueda T, Takatori H, Nishino R, Kaneko S. Different signaling pathways in the livers of patients with chronic hepatitis B or chronic hepatitis C. Hepatology. 2006;44(5):1122-1138.

21. Mcgaughan GW, Shackel NA, Gorrell MD. Discussion on differential gene expression between chronic hepatitis $\mathrm{B}$ and $\mathrm{C}$ hepatic lesion. Gastroenterology. 2001;121(5):1263-1264.

22. Neuveut C, Wei Y, Buendia MA. Mechanisms of HBV-related hepatocarcinogenesis. J Hepatol. 2010;52(4):594-604.

23. Tanaka M, Tanaka H, Tsukuma H, Ioka A, Oshima A, Nakahara T. Risk factors for intrahepatic cholangiocarcinoma: a possible role of hepatitis B virus. J Viral Hepat. 2010;17(10):742-748.

24. Choi J, Ghoz HM, Peeraphatdit T, et al. Aspirin use and the risk of cholangiocarcinoma. Hepatology. 2016;64(3):785-796.

25. Palmer WC, Patel T. Are common factors involved in the pathogenesis of primary liver cancers? A meta-analysis of risk factors for intrahepatic cholangiocarcinoma. J Hepatol. 2012;57(1):69-76.

26. Marchesini G, Bugianesi E, Forlani G, et al. Nonalcoholic fatty liver, steatohepatitis, and the metabolic syndrome. Hepatology. 2003;37(4):917-923.

27. Hamaguchi M, Kojima T, Takeda N, et al. The metabolic syndrome as a predictor of nonalcoholic fatty liver disease. Ann Intern Med. 2005;143(10):722-728.

28. Adams LA, Lymp JF, St Sauver J, et al. The natural history of nonalcoholic fatty liver disease: a population-based cohort study. Gastroenterology. 2005;129(1):113-121.

29. Starley BQ, Calcagno CJ, Harrison SA. Nonalcoholic fatty liver disease and hepatocellular carcinoma: a weighty connection. Hepatology. 2010;51(5):1820-1832.

30. Welzel TM, Graubard BI, Zeuzem S, El-Serag HB, Davila JA, Mcglynn KA. Metabolic syndrome increases the risk of primary liver cancer in the United States: a study in the SEER-Medicare database. Hepatology. 2011;54(2):463-471.

31. Farrell GC, Larter CZ. Nonalcoholic fatty liver disease: from steatosis to cirrhosis. Hepatology. 2006;43(2 Suppl 1):S99-S112. 
Cancer Management and Research

\section{Publish your work in this journal}

Cancer Management and Research is an international, peer-reviewed open access journal focusing on cancer research and the optimal use of preventative and integrated treatment interventions to achieve improved outcomes, enhanced survival and quality of life for the cancer patient

The manuscript management system is completely online and includes

Submit your manuscript here: https://www.dovepress.com/cancer-management-and-research-journal

a very quick and fair peer-review system, which is all easy to use. Visit $\mathrm{http}: / / \mathrm{www}$.dovepress.com/testimonials.php to read real quotes from published authors. 